\section{Gefahrenmelder im Bronchialepithel}

Das NLRP3-Inflammasom ist ein Proteinkomplex, der in Zellen der angeborenen Immunabwehr vorkommt und dessen Aktivierung die Produktion des Zytokins Interleukin-1 $\beta$ in Gang setzt. Nicht geklärt war bisher, ob das Inflammasom auch in den Epithelzellen der oberen Atemwege exprimiert wird.

$\mathrm{M}$ ithilfe serieller Färbungen menschlichen Bronchialgewebes und anschließendem Immunoblot gelang es kanadischen Forschern zunächst die Expression sowohl des NLRP3-Inflammasoms als auch des Enzyms Caspase-1, was für die IL-1 $\beta$-Aktivierung notwendig ist, in den Atemwegsepithelien nachzuweisen. In einem weiteren Experiment setzte das Team den menschlichen Epithelzellen Feinstaub mit einem Endotoxingehalt von $6,4 \mathrm{EU} / \mathrm{ml}$ zu: Dies führte zu einem signifikanten Anstieg der IL-1 $\beta$-Produktion. Um die Funktionalität des Inflammasoms (das Akronym NLRP3 steht für Nucleotide-binding domain and Leucine-rich Repeat Protein 3) zu messen, applizierten die Kanadier zum einen den Sulfonylharnstoff Glibenclamid, der in der Lage ist, das NLRP3-Inflammasom unspezi- fisch zu hemmen, sowie den spezifischen Caspase-1-Inhibitor Z-YVAD-FMK. Beide Substanzen führten zu einer deutlich abgeschwächten Freisetzung von IL-1 $\beta$ nach der Feinstaubexposition.

Die entscheidende Rolle des Inflammasoms bei der Immunantwort auf die Feinstaubtoxine bestätigte sich in In-vivoExperimenten: Bei Wildtyp-Mäusen mit aktivem NLRP3-Komplex führten die Schadstoffe zu signifikanten Anstiegen der IL-1 $\beta$-Konzentrationen in der bronchoalveolären Lavage. Knockout-Mäuse, bei denen das Inflammasom ausgeschaltet war, zeigten diese Veränderungen nicht.

Die IL-1 $\beta$-Produktion ließ sich auch im Lungenepithel der Wildtyp-Mäuse nachweisen, nicht aber bei den Mäusen mit ausgeschaltetem NLRP3-Komplex. Und schließlich zeigte sich, dass auch feinstaubinduzierte Veränderungen im Phänotyp dendritischer Zellen offenbar über das Inflammasom vermittelt werden.

Fazit: Die Ergebnisse zeigen dreierlei: Das NLRP3-Inflammasom wird im Epithel der oberen Atemwege exprimiert und durch Feinstaubexposition aktiviert, was über das Enzym Caspase-1 zur Freisetzung von IL-1 $\beta$ führt. Zum zweiten vermittelt das Inflammasom eine Entzündungsreaktion mit Neutrophilie. Und schließlich ist der NLRP3-Komplex auch an adaptiven Veränderungen dendritischer Zellen beteiligt. Mit der Hemmung des Inflammasoms konnten die Forscher Entzündungsreaktionen unterbinden. Diese Erkenntnis könnte nach Hirota und Kollegen die Grundlage für zukünftige therapeutische Ansätze liefern. Dr. Elke Oberhofer

Hirota JA et al. The airway epithelium nucleotide-binding domain and leucine-rich repeat protein 3 inflammasome is activated by urban particulate matter. J Allergy Clin Immunol 2012; 129: 1116-25.e6 